\title{
Cognitive Function in the Prefrailty and Frailty Syndrome
}

\author{
Deirdre A. Robertson, MSc, * George M. Savva, PhD, ${ }^{\dagger}$ Robert F. Coen, PhD, ${ }^{*}$ and \\ Rose-Anne Kenny, $M D^{*}$
}

OBJECTIVES: To explore the relationship between cognitive function and frailty.

DESIGN: A cross-sectional study using data from Wave 1 of The Irish Longitudinal Study on Ageing, a population representative study of adults aged 50 and older in the Republic of Ireland.

SETTING: Community-dwelling adults completed a home- or health center-based nurse-led assessment.

PARTICIPANTS: Individuals aged 50 and older without a history of stroke, Parkinson's disease, or severe cognitive impairment (Mini-Mental State Examination (MMSE) score $<18)$ and not taking antidepressants $(\mathrm{N}=4,649)$.

MEASUREMENTS: A cognitive battery including MMSE, Montreal Cognitive Assessment, Color Trails Test, Cambridge Mental Disorders of the Elderly Examination memory and executive function subtests, 10-word recall, Sustained Attention to Response Task, and choice reaction time was used to generate composite scores of cognitive domains. Frailty was assessed according to weakness, slowness, exhaustion, low physical activity, and weight loss.

RESULTS: After full adjustment, cognitive function across all domains except self-rated memory and processing speed was significantly worse in prefrail and frail participants $(P<.05)$ than in those who were robust. Weakness and walking speed were most consistently linked to poorer cognition, whereas low activity and weight loss were not independently associated with any cognitive domain. Exhaustion was associated with global cognition ( $B=-0.18 \pm 0.06)$, with some evidence of links to objectively measured and self-rated memory.

CONCLUSION: Cognitive function is worse across multiple cognitive domains in prefrail and frail individuals aged 50 and older than in those who are robust, although the

From the *The Irish Longitudinal Study on Ageing (TILDA), Department of Medical Gerontology, Trinity College Dublin, Ireland; 'ंSchool of Health Sciences, University of East Anglia, Norwich Research Park, Norwich, Norfolk, UK; ${ }^{\ddagger}$ Mercer's Institute for Research on Ageing, St James's Hospital, Dublin, Ireland; and \$Institute of Neuroscience, Trinity College, Dublin, Ireland.

Address correspondence to Deirdre Robertson. TILDA, Chemistry Extension Building, Lincoln Gate, Trinity College, Dublin 2, Ireland. E-mail: droberts@tcd.ie

DOI: 10.1111 /jgs.13111 absolute differences are small after adjusting for confounding factors. J Am Geriatr Soc 62:2118-2124, 2014.

Key words: prefrailty; frailty; cognition; dementia

A lthough frailty is a relatively newly defined concept, it has recently been suggested that it should be termed as one of the "geriatric giants" that define geriatric medical practice. ${ }^{1}$ Frailty is characterized by the body's inability to respond adequately to external stressors and a greater risk of adverse outcomes, including disability, hospitalization, institutionalization, and death, ${ }^{2}$ but the precursors to frailty and the associations between frailty and other geriatric syndromes are still relatively unknown. One of these questions is to what extent frailty is associated with another geriatric giant, cognitive impairment, and the different domains of cognitive function.

A number of cross-sectional and longitudinal studies have shown that frail people are more likely to have dementia or cognitive impairment and that those who already have dementia are more likely to be frail..$^{3-10}$ This raises important questions about frailty as a potentially modifiable risk factor for dementia and vice versa. Furthermore, frailty and cognitive impairment share a number of common risk factors, including cardiovascular disease and depression. ${ }^{11-14} \mathrm{~A}$ recent study addressed some of these risk factors by engaging frail and nonfrail participants in an intensive exercise program over 12 weeks. ${ }^{15}$ Nonfrail and frail participants showed an increase in cognitive ability, physical function, and quality of life, raising the possibility that interventions might delay or reverse frailty and cognitive impairment. ${ }^{15,16}$

Although many studies have analyzed the relationship between frailty and cognition, most have focused on dementia or change in global cognition as measured using the Mini-Mental State Examination (MMSE). ${ }^{17-21}$ Few have examined the association between frailty and individual cognitive domains, and in those that have, the results have been inconsistent. The Rush Memory and 
Aging study found that frailty was associated with a faster rate of decline in cognitive scores across all six domains measured, including memory. ${ }^{21}$ Other studies have suggested that memory is less affected than executive function, ${ }^{19,20}$ and consistent with this finding, three prospective studies have shown that frailty is more strongly associated with vascular than Alzheimer's type dementia. ${ }^{7,17,20,22}$ To understand why there is an association between these two syndromes, it is necessary to further explore the relationship between frailty, individual frailty indicators, and specific cognitive domains. Understanding the relationship may also indicate possible causal pathways that can be targeted using interventions. Furthermore, few studies have examined cognitive performance in prefrailty as well as frailty.

Previous studies on this topic have used convenience samples or have restricted their analysis to specific populations such as low socioeconomic groups that are not generalizable to other populations. ${ }^{17,19-21}$ The current study examined the association between frailty and cognitive function in a large population-representative sample of adults aged 50 and older. Middle-aged and older participants were analyzed independently and together, allowing comparison of the association between frailty and cognition across a broad range of age and severity of frailty, including those in the early (prefrail) stages.

\section{METHODS}

\section{Population}

Data were taken from Wave 1 of The Irish Longitudinal Study on Ageing (TILDA), a prospective study of a sample representative of the population of the Republic of Ireland aged 50 and older living in the community (not in longterm care facilities). Participants who reported a doctor's diagnosis of dementia or who were unable to consent personally to participation because of severe cognitive impairment (judged at an interviewer's discretion) were not included in Wave 1 of the study. The full cohort and assessment has been described in detail elsewhere, ${ }^{23}$ but in brief, 8,175 participants aged 50 and older were interviewed in their own homes and then underwent a nurseadministered health assessment at one of two dedicated health centers. Participants unable or unwilling to attend a health assessment center received a modified nurse-delivered health assessment at home. The response rate to the interview was $62.0 \%$, and $61.6 \%$ of participants $(\mathrm{n}=6,150)$ completed the health $(\mathrm{n}=5,274)$ or home $(\mathrm{n}=876)$ assessment.

Data from participants who had participated in a full health center assessment that included all of the physical and cognitive items needed to compose a measure of frailty together with the battery of neuropsychological measures were used.

Consistent with the definition of frailty by Fried and colleagues, participants who had Parkinson's disease $(\mathrm{n}=16)$ or a history of stroke $(\mathrm{n}=67)$; those taking antidepressants $(\mathrm{n}=284)$, dementia medication $(\mathrm{n}=6)$, or levodopa and carbidopa $(\mathrm{n}=1)$; and those with severe cognitive impairment (MMSE score $<18 ; \mathrm{n}=11$ ) were excluded. ${ }^{24}$

\section{Assessment of Frailty}

The Fried phenotypic definition of frailty was used because it incorporates a definition of prefrailty and frailty. ${ }^{24}$ Participants were classified as frail if they met three of the following five criteria: poor grip strength, slow gait speed, low levels of physical activity, unintentional weight loss, and exhaustion. ${ }^{24}$ Poor grip strength was assessed using a dynamometer. Two readings from the dominant hand were taken, and mean strength calculated. The 20th percentile was calculated, and populationspecific cutoffs were derived after adjustment for age, sex, and body mass index. Gait speed was measured using the GAITRite portable electronic walkway system (CIR Systems, Inc., Havertown, PA). Participants walked at their usual pace along a $4.88-\mathrm{m}$ (16-foot) walkway with an extra $2.5 \mathrm{~m}$ at each end to allow for acceleration and deceleration. Mean gait speed was calculated from two walks. Participants who fell below the sex- and heightadjusted 20th percentile were considered to have slow gait speed, as defined according to the Fried methodology. ${ }^{24}$ Physical activity was measured using the short form of the International Physical Activity Questionnaire, which converts levels of physical activity in various domains into predicted kilocalories expended per week. ${ }^{25}$ Unintentional weight loss was assessed using the question, "In the past year, have you lost 10 pounds $(4.5 \mathrm{~kg})$ or more in weight when you were not trying to." Exhaustion was assessed using two items from the 20-item Center for Epidemiologic Studies Depression Scale (CES-D): "I could not get going," and "I felt that everything I did was an effort," with four possible responses to each question: never, rarely, sometimes, often. A response of sometimes or often to either question was classified as exhaustion. ${ }^{26}$ Participants with three or more indicators were defined as frail, those with one or more as prefrail, and those with none as robust. ${ }^{24}$

\section{Assessment of Cognitive Function}

The cognitive battery included tests of global cognition, attention, memory, executive function, and speed of processing. Composite scores were calculated for each of these domains. Although each of the tests measures more than one domain of cognitive function, they were classified based on the main cognitive component involved.

Global cognition was assessed using the MMSE and Montreal Cognitive Assessment (MoCA). ${ }^{27,28}$ Attention was assessed according to two tasks: part 1 of the Color Trails Test and the Sustained Attention to Response Task (SART). ${ }^{29,30}$ The Color Trails Test involves two parts; the first requires participants to draw a line connecting circles numbered 1 to 25 in consecutive order. The second part requires connecting numbered circles while alternating between pink and yellow circles (e.g., pink 1, yellow 2, pink 3). The first part assesses visual scanning and attention. The mental flexibility involved in the switching task of part 2 assesses executive function. The SART tests the ability to sustain attention for long periods of time during a repetitive task. Participants were shown a computer screen that repeated a sequence of numbers from 1 to 9 for approximately 4 minutes. Numbers appeared every 
$300 \mathrm{~ms}$, and participants were asked to click in response to each number except the number 3 .

Memory was assessed using three methods: the Picture Recognition and Recall subtests of the Cambridge Mental Disorders of the Elderly Examination (CAMDEX), a 10word immediate and delayed recall test using a list of 10 words previously developed for the Health and Retirement Survey, ${ }^{31,32}$ and self-rated memory measured using the question: "How would you rate your day-to-day memory at the present time? Would you say it is: excellent, very good, good, fair, or poor?"

Executive function was assessed using three tasks: the Visual Reasoning subtest of the CAMDEX (participants are asked to identify which of six objects would complete a pattern of three similar objects), part 2 of the Color Trails Test, and the MoCA verbal fluency subtest (participants were given 1 minute to name as many words beginning with $F$ as they could think of). ${ }^{28,29,31}$

Processing speed was assessed using a computer-based program. Participants were instructed to press a central button on the keyboard and wait for a stimulus (yes/no) to appear on screen. When the stimulus appeared participants released the central button and pressed one of two other buttons labeled "yes" or "no" to correspond to the stimulus on screen. The full task involves approximately 100 repetitions. Cognitive processing speed is measured as the time taken to release the first button on presentation of the stimulus.

Composite scores for each cognitive domain were calculated by obtaining $z$-scores for each cognitive test and combining them to create total scores for global cognition (MoCA and MMSE), executive function (visual reasoning, Color Trail Test part 2, MoCA "F letter" subtest), memory (visual recall, visual recognition, immediate memory, delayed memory), attention (Color Trail Test part 1 and SART), and speed of processing (Choice Reaction Time). The standardized versions of these scores constituted the composite cognitive score for each domain. Using $Z$ scores allows the tests to be combined, which leads to more-reliable estimates of the effects on each domain, reduces the number of independent tests being conducted, and allows the predictive value of frailty indicators for each domain of cognitive function to be compared in a standardized way. These lead to estimates of effects for each domain in the population, which can be approximately related back to differences in individual tests using the population standard deviations for each test.

\section{Covariates and Exclusion Criteria}

Education was categorized as primary and below, secondary, or tertiary and above. History of stroke and chronic conditions, including joint problems, cataracts, glaucoma, age-related macular degeneration, lung disease, asthma, arthritis, osteoporosis, any type of cancer, Parkinson's disease, peptic ulcer, liver disease, varicose ulcer, alcohol or substance abuse, chronic pain, and incontinence, were ascertained according to self-report of a doctor's diagnosis. Height and weight were measured and used to calculate sex-adjusted cutoffs for the frailty measures. Participants were also asked to record all of the medications taken on a regular basis, and when possible, interviewers asked to see the packages to confirm.

\section{Statistical Analyses}

Sociodemographic characteristics of the three groups (frail, prefrail, robust) were compared. The relationship between frailty and each of the individual cognitive tests was then assessed using linear regression models.

Owing to large differences in the mean age of each frailty group, it was not deemed useful to compare cognitive test scores between groups without taking demographic factors into account. Therefore, for descriptive purposes, the marginal mean score of each cognitive test was calculated for each frailty group using regression models adjusted for age (as a continuous variable, including an age-squared term to account for nonlinear relationship between cognitive function and age), sex, and educational attainment.

Regression models were then used to estimate the association between frailty and prefrailty and each cognitive domain as measured according to composite $z$-scores, and these were plotted to compare the relative effect of frailty on each cognitive domain. This analysis was adjusted for age, age squared, sex, education, all chronic conditions reported, and number of regular medications. Beta coefficients from these analyses indicate differences in cognitive scores between frailty groups in terms of that standard deviation of that cognitive domain in the population.

Finally, the effect of each frailty indicator on each domain of cognitive function was assessed using separate models, with the composite cognitive scores as an outcome variable and all frailty indicators as binary variables adjusted for age, age squared, sex, education, chronic conditions, and medications, as above. For models including individual indicators of frailty, an outcome was considered statistically significant at $P<.008(.05 / 6)$, to avoid potential false positives caused by the large number of hypothesis tests.

Analyses were performed using Stata version 12.1 (StataCorp LP, College Station, TX).

\section{RESULTS}

Of 4,649 people aged 50 and over included in the analysis, $90(1.9 \%)$ were frail, and $1,444(31.1 \%)$ were prefrail. The prevalence of each frailty indicator in the population was roughly similar (exhaustion $=7.8 \%$, weight loss $=5.6 \%$, slowness $=8.6 \%$, weakness $=8.9 \%$ ), although low physical activity had a higher prevalence of $14.2 \%$. The most common combination of frailty indicators in the frail sample was slow walking speed, poor grip strength, and low levels of physical activity (24.4\% of frail sample), followed by walking speed, grip, and exhaustion (15.6\%). Frail participants were significantly older (mean age $69.3 \pm 11.1$ ) than prefrail $(63.8 \pm 9.1)$ and robust $(60.7 \pm 7.7)$ participants $\left(F_{2,4638}=111.1, P<.001\right)$ and significantly less likely to have completed the third-level education $(18.9 \%$ of frail participants, $33.1 \%$ of prefrail, $39.3 \%$ of robust; chi-square $(4)=51.4, P<.001)$. Although a greater proportion of frail participants were female $(57.8 \%$ vs $53.9 \%$ 
prefrail, $53.1 \%$ robust), this was not statistically significant (chi-square $(2)=.95, P=.62$ ). (See Table 1 for full demographic characteristics.)

An analysis of performance in each cognitive measure indicated that frail participants achieved significantly lower scores on the majority of cognitive tests after accounting for age, sex, and education. Table 2 shows the sample and marginal means for each cognitive test after standardizing to the sample average age, sex, and educational attainment. For example, the mean MMSE score \pm standard deviation was $27.6 \pm 2.2$ for frail participants and $28.8 \pm 1.5$ for robust participants. The marginal means adjusted for age, sex, and education remained statistically significantly different, although the difference was smaller than that indicated by the unadjusted sample means. For example, the marginal mean MMSE (standard error (SE)) was $28.0(0.2)$ for frail participants and 28.7 (0.03) for robust participants. There was no significant difference between frail and robust participants on only two tests: visual recall and visual recognition.

Using composite scores representing the six cognitive domains - global cognition, executive function, memory, attention, processing speed, self-rated memory-linear regression was used to determine whether being prefrail or frail significantly predicted poorer scores in each cognitive domain. Figure 1 indicates a dose-response relationship whereby prefrail participants scored statistically significantly less well than robust participants, and frail participants scored significantly lower than prefrail participants on tests of global cognitive function, executive function, memory, and attention, after adjusting for all other covariates. Processing speed and self-rated memory were not significantly worse in frail participants, although the direction of the observed effect was similar to that in the other cognitive domains. For example, prefrail participants had an average global cognitive function score $0.14 \pm 0.03$ points lower than that of robust participants, and frail participants had an average global cognitive function score $0.43 \pm 0.03$ points lower than that of robust participants. This corresponds well to the differences seen in individual test scores adjusted for demographic differences (Table 2). For example, there is a marginal mean difference in MoCA score of 0.6 points between prefrail and robust participants and a difference of 1.3 points between frail and robust participants (with the population standard deviation for MoCA approximately 3 points).

The same analysis was conducted separately for each of three age categories $(50-64,65-74, \geq 75)$. Although a similar relationship was found between frailty and global cognitive function in each age group, executive function was associated with frailty only in the group aged 75 and older $(B=-0.37, \mathrm{SE}=0.16, P=.03)$, and memory was associated with frailty only in the group aged 50 to 64 $(B=-0.35, \mathrm{SE}=0.13, P=.005)$. Nevertheless, the numbers with frailty were small when broken into age categories $(\mathrm{n}=34,20$, and 36 in each age category, respectively), so care must be taken in interpreting these results. (Full results reported in Appendix S1.)

Finally, the score for each cognitive domain was regressed on the five individual frailty indicators simultaneously after adjusting for all covariates, with the cut off for statistical significance of $P<.008$ used to account for multiple testing. Processing speed, attention, and executive function were statistically significantly poorer in participants with slow gait speed. Executive function was also lower in participants with poor grip strength. Objectively measured and self-rated memory were not statistically significantly associated with any specific frailty indicator at the $P<.008$ level (Table 3). Weight loss and low levels of physical activity were not independently associated with any cognitive domain.

\section{DISCUSSION}

Middle-aged and older participants of a large populationrepresentative sample who were prefrail or frail had statistically significantly poorer performance in multiple domains of cognitive function than their robust peers, after adjusting for age, sex, educational attainment, chronic conditions, and number of medications and excluding those with stroke, severe cognitive impairment, or antidepressant use. Although the absolute difference in mean cognitive function between the groups after adjusting for confounders was small, the dose-response relationship between increasing levels of frailty and poorer cognitive function supports the association, and small differences in cognitive scores could be considered substantial with respect to the variability of cognitive tests in this population. For instance, although the absolute differences in global test scores between the frail and robust participants were small in clinical terms $(\sim 1$ point in each case), they represent changes of approximately 0.4 standard deviations with respect to the distribution of scores in this young, healthy population.

Conducting the analysis stratified according to age group $(50-64,65-74, \geq 75)$ suggested that attention may

\section{Table 1. Characteristics of Nonfrail, Frail, and Robust Community-Dwelling Participants}

\begin{tabular}{lcccc}
\hline \multicolumn{1}{c}{ Characteristic } & Robust, $\mathbf{n}=\mathbf{3 , 1 1 5}$ & Prefrail, $\mathbf{n}=\mathbf{1 , 4 4 4}$ & Frail, $\mathbf{n}=\mathbf{9 0}$ & Total, $\mathbf{N}=\mathbf{4 , 6 5 1}$ \\
\hline Age, mean \pm SD & $60.7 \pm 7.7$ & $63.8 \pm 9.1$ & $69.3 \pm 11.1^{\mathrm{a}}$ & $61.81 \pm 8.4$ \\
Female, \% & 53.1 & 53.9 & 57.8 & 53.5 \\
Third-level education, \% & 39.3 & 33.1 & $18.9^{\mathrm{a}}$ & 36.9 \\
Mini-Mental State Examination & $28.8 \pm 1.5$ & $28.4 \pm 1.8$ & $27.6 \pm 2.2^{\mathrm{a}}$ & $28.7 \pm 1.7$ \\
Score, mean \pm SD & $1.8 \pm 2.1$ & $2.8 \pm 2.8$ & $5.1 \pm 3.1^{\mathrm{a}}$ & $2.2 \pm 2.4$ \\
Number of medications, mean $\pm \mathrm{SD}$ & & &
\end{tabular}

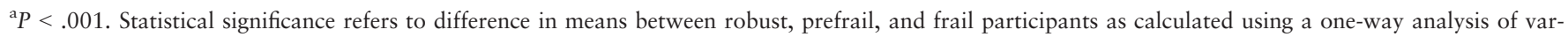
iance.

$\mathrm{SD}=$ standard deviation. 
Table 2. Sample Mean and Adjusted Marginal Mean Scores for Individual Cognitive Measures for Each Frailty Category

\begin{tabular}{|c|c|c|c|c|c|c|}
\hline \multirow[b]{2}{*}{ Cognitive Test (Range) } & \multicolumn{3}{|c|}{ Sample Mean \pm Standard Deviation } & \multicolumn{3}{|c|}{$\begin{array}{l}\text { Marginal Mean (Standard Error) } \\
\text { Standardized to Population Average Age, } \\
\text { Sex, and Educational Attainment }\end{array}$} \\
\hline & $\begin{array}{l}\text { Robust, } \\
\mathrm{n}=3,115\end{array}$ & $\begin{array}{l}\text { Prefrail, } \\
n=1,444\end{array}$ & $\begin{array}{l}\text { Frail, } \\
\mathrm{n}=90\end{array}$ & $\begin{array}{l}\text { Robust, } \\
n=3,115\end{array}$ & $\begin{array}{c}\text { Prefrail, } \\
n=1,444\end{array}$ & $\begin{array}{l}\text { Frail, } \\
\mathrm{n}=90\end{array}$ \\
\hline \multicolumn{7}{|l|}{ Global cognition } \\
\hline $\begin{array}{l}\text { Mini-Mental State Examination } \\
\text { score }(0-30)^{\mathrm{a}}\end{array}$ & $28.8 \pm 1.5$ & $28.4 \pm 1.8$ & $27.6 \pm 2.2$ & & $28.6(0.04)^{\dagger}$ & $28.0(0.2)^{\dagger}$ \\
\hline $\begin{array}{l}\text { Montreal Cognitive } \\
\text { Assessment }(0-30)^{\mathrm{a}}\end{array}$ & $25.6 \pm 3.0$ & $24.7 \pm 3.5$ & $23.2 \pm 3.7$ & $25.5(0.05)$ & $24.9(0.08)^{\dagger}$ & $24.2(0.4)^{f}$ \\
\hline \multicolumn{7}{|l|}{ Executive function } \\
\hline Visual reasoning $(0-6)^{\mathrm{a}}$ & $3.2 \pm 1.3$ & $3.0 \pm 1.3$ & $2.5 \pm 1.3$ & $3.2(0.02)$ & $3.1(0.03)^{d}$ & $2.8(0.1)^{\mathrm{e}}$ \\
\hline Color Trails Test $\mathrm{B}$, seconds ${ }^{\mathrm{b}}$ & $103.0 \pm 35.1$ & $115.9 \pm 42.5$ & $139.1 \pm 58.9$ & $105.7(0.6)$ & $111.4(0.9)^{f^{\prime}}$ & $119.7(3.7)^{\mathrm{e}}$ \\
\hline Verbal fluency $^{\mathrm{a}}$ & $12.6 \pm 5.0$ & $11.7 \pm 5.0$ & $9.8 \pm 4.5$ & $12.5(0.08)$ & $11.9(0.1)^{\dagger}$ & $10.7(0.5)^{\dagger}$ \\
\hline \multicolumn{7}{|l|}{ Memory } \\
\hline Visual recall $(0-6)^{\mathrm{a}}$ & $3.3 \pm 1.1$ & $3.2 \pm 1.1$ & $2.9 \pm 1.2$ & $3.3(0.02)$ & $3.2(0.03)^{d}$ & $3.2(0.1)$ \\
\hline Visual recognition $(0-6)^{\mathrm{a}}$ & $5.7 \pm 0.6$ & $5.6 \pm 0.7$ & $5.5 \pm 0.8$ & $5.7(0.01)$ & $5.6(0.02)^{d}$ & $5.6(0.07)$ \\
\hline Immediate $(0-10)^{\mathrm{a}}$ & $6.1 \pm 1.6$ & $5.8 \pm 1.6$ & $5.0 \pm 1.5$ & $6.1(0.03)$ & $5.9(0.04)^{\mathrm{e}}$ & $5.5(0.2)^{f^{\prime}}$ \\
\hline Delayed $(0-10)^{\mathrm{a}}$ & $6.5 \pm 2.2$ & $6.0 \pm 2.2$ & $4.9 \pm 2.2$ & $6.4(0.04)$ & $6.2(0.05)^{\mathrm{e}}$ & $5.6(0.2)^{\mathrm{e}}$ \\
\hline Self-rated $(0-5)^{b, c}$ & $2.4 \pm 0.9$ & $2.6 \pm 0.9$ & $2.9 \pm 0.9$ & $2.5(0.02)$ & $2.6(0.02)^{\dagger}$ & $2.8(0.1)^{\mathrm{e}}$ \\
\hline \multicolumn{7}{|l|}{ Attention } \\
\hline Color Trails Test $\mathrm{A}$, seconds ${ }^{\mathrm{b}}$ & $51.4 \pm 20.7$ & $59.5 \pm 27.5$ & $78.6 \pm 43.2$ & $53.1(0.4)$ & $56.7(0.6)^{\dagger}$ & $66.7(2.3)^{\mathrm{e}}$ \\
\hline \multicolumn{7}{|c|}{ Sustained Attention to Response Task } \\
\hline Mean time, seconds ${ }^{b}$ & $370.3 \pm 95.5$ & $393.6 \pm 103.6$ & $428.8 \pm 105.5$ & $372.9(1.8)$ & $388.9(2.6)^{f}$ & $410.7(10.9)^{\mathrm{e}}$ \\
\hline Omission errors $(0-200)^{b}$ & $5.9 \pm 8.1$ & $8.7 \pm 11.2$ & $13.5 \pm 16.3$ & $6.4(0.2)$ & $7.9(0.2)^{f}$ & $10.2(1.0)^{d}$ \\
\hline Commission errors $(0-25)^{b}$ & $3.4 \pm 3.5$ & $4.5 \pm 4.3$ & $5.6 \pm 4.6$ & $3.6(0.07)$ & $4.1(0.1)^{\dagger}$ & $4.2(0.4)$ \\
\hline \multicolumn{7}{|l|}{ Processing speed } \\
\hline Cognitive reaction time, $\mathrm{ms}^{\mathrm{b}}$ & $489.9 \pm 91.6$ & $505.9 \pm 100.1$ & $550.3 \pm 134.3$ & $492.9(1.7)$ & $500.2(2.5)^{d}$ & $529.2(10.4)^{c}$ \\
\hline
\end{tabular}

Marginal means were estimated using a regression model and standardized to the population average age, sex, and educational attainment such that the confounding effect of these factors was removed.

${ }^{\mathrm{a}}$ Higher scores indicate better cognitive function.

${ }^{b}$ Lower scores indicate better cognitive function.

${ }^{c} 1$ = excellent, 2 = very good, 3 = good, $4=$ fair, 5 = poor.

Statistically significant difference from robust group: $P<{ }^{\mathrm{d}} .05,{ }^{\mathrm{e}} .01,{ }^{\mathrm{f}} .001$.

be associated with frailty only in those aged 75 and older, whereas memory may be associated with frailty only in those aged 50 to 65 , although the numbers of frail participants in these groups were small, so the results should be interpreted with caution. Individual indicators of frailty were also independently associated with cognitive function. Processing speed, executive function, and attention were associated with weak grip strength and slow walking speed, but memory and self-rated memory were not independently associated with the indicators of frailty after adjustment for chronic conditions and medication.

These findings contradict some previous studies suggesting that frailty is not significantly associated with poor memory ${ }^{17,19,20}$ but concur with findings from the Rush Memory and Aging study, which found that higher levels of frailty were associated with a faster rate of decline in all cognitive domains. ${ }^{21}$ This discrepancy may have occurred because of the small or homogeneous samples in previous studies. ${ }^{17,19,20}$

Previous work has suggested that only slow gait speed and weak grip strength are associated with greater risk of developing mild cognitive impairment (MCI) or non-Alzheimer's disease dementia. ${ }^{5,21}$ The current results may support the latter findings to some extent because gait speed and grip strength were associated with lower executive function, one of the hallmark cognitive domains of nonAlzheimer's disease dementia.

Several mechanisms have been suggested as mediators in the relationship between frailty and cognition. These include biological and psychological factors, including neuropathology, cardiovascular disease, inflammation, hormonal changes, nutrition, social isolation, and social vulnerability. ${ }^{10} \mathrm{~A}$ recent review on the topic of cognitive impairment and frailty outlined a hypothetical model describing how these mechanisms interact with each other to influence the cycle of frailty and cognition. ${ }^{10}$ The results of the current study suggest that the cognitive part of the model could be further refined because not all cognitive domains may become impaired simultaneously but may become impaired depending on the age and frailty indicators present. For example, a person with slow walking speed may have poor executive function specifically but not poor memory. This is interesting in light of previous work on gait speed and cognition that found that both were associated with smaller volume of the prefrontal area of the brain. ${ }^{33}$ It is possible that the etiology of frailty and cognitive decline may differ depending on which indicators of frailty are present. 


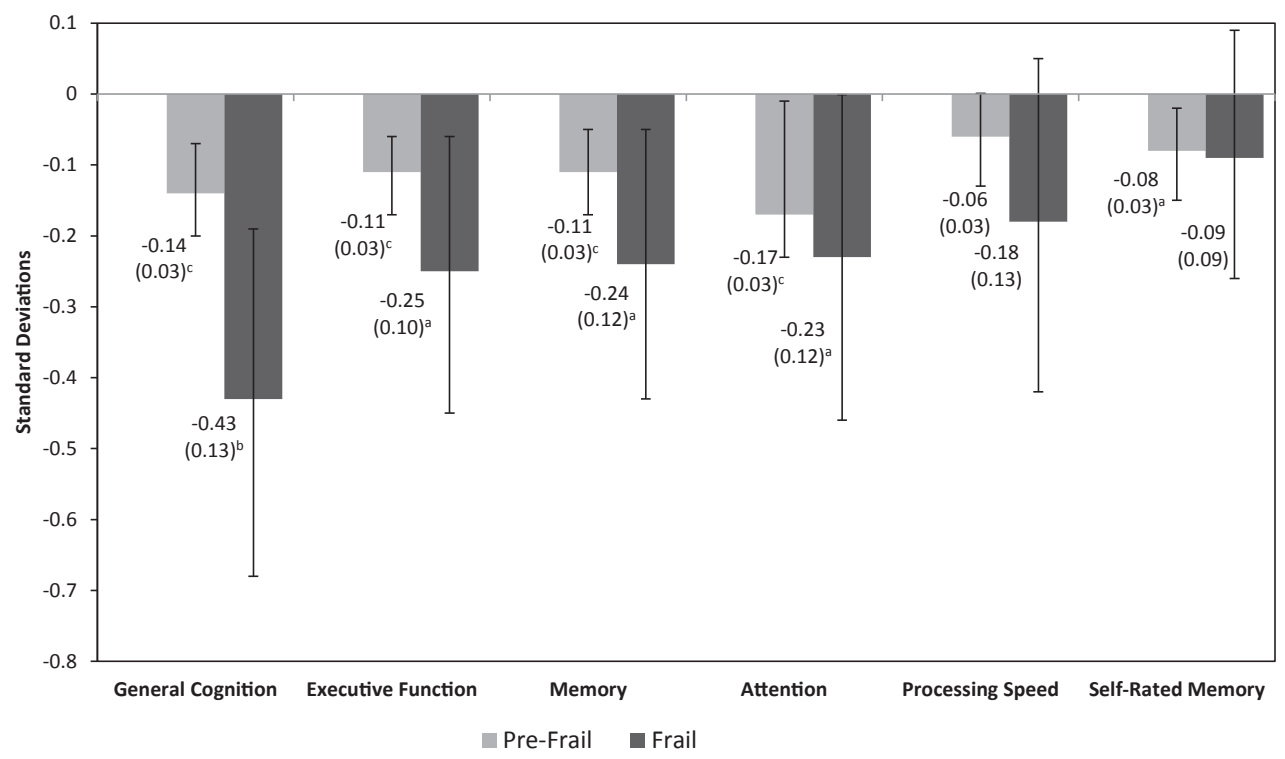

Figure 1. Z-scores (with standard errors) for cognitive domains in prefrail and frail older adults compared with robust, after adjusting for age, sex, education, chronic conditions, and medication use. $P<<^{\mathrm{a}} .05,{ }^{\mathrm{b}} .01,{ }^{\mathrm{c}} .001$. Showing beta coefficients (standard errors) and $95 \%$ confidence intervals. Robust group compared with prefrail and frail. Z-scores were standardized such that the mean domain score of robust individuals was 0 , with the score indicating the difference in terms of standard deviation of composite cognitive domain scores. For example, frail individuals scored 0.43 standard deviations less than robust individuals on global cognitive tests, after adjusting for covariates.

Table 3. Results of a Multivariate Linear Regression Estimating the Independent Effect of Individual Components of the Frailty Syndrome on Standardized Composite Domains of Cognitive Function

Global Cognition Executive Function Memory Attention Processing Speed Self-Rated Memory

Frailty Component

Beta Coefficient (Standard Error)

\begin{tabular}{llllrrr}
\hline Exhaustion & $-0.18(0.06)^{\mathrm{a}}$ & $-0.04(0.05)$ & $-0.14(0.06)$ & $-0.02(0.06)$ & $-0.02(0.06)$ & $-0.13(0.06)$ \\
Weight loss & $-0.07(0.06)$ & $-0.01(0.06)$ & $-0.02(0.06)$ & $-0.02(0.07)$ & $0.02(0.07)$ & $0.02(0.06)$ \\
Activity & $-0.08(0.04)$ & $-0.04(0.04)$ & $-0.09(0.04)$ & $-0.09(0.04)$ & $-0.01(0.04)$ & $-0.09(0.04)$ \\
Slow gait & $-0.11(0.06)$ & $-0.20(0.05)^{\mathrm{a}}$ & $-0.13(0.06)$ & $-0.25(0.05)^{\mathrm{a}}$ & $-0.16(0.06)^{\mathrm{a}}$ & $0.06(0.05)$ \\
Weak grip & $-0.26(0.06)^{\mathrm{a}}$ & $-0.14(0.05)^{\mathrm{a}}$ & $-0.09(0.05)$ & $-0.14(0.05)$ & $-0.14(0.05)$ & $-0.09(0.05)$ \\
\hline
\end{tabular}

Beta coefficients indicate the independent effect of each frailty indicator on each cognitive domain. For example, individuals with self-reported exhaustion scored 0.18 standard deviations less on tests of global cognitive function after other frailty indicators were taken into account $(P<.008)$.

${ }^{\mathrm{a}} P<.008$. Adjusted for age, age-squared, sex, level of education, chronic conditions, and number of medications.

The strength of this study lies in its comprehensive, nurse-led health assessment that included an extensive battery of cognitive tests and objective assessments of gait speed and grip strength. The current body of knowledge of the link between frailty and specific cognitive domains is limited to a small number of studies. ${ }^{10}$ The main limitation is the cross-sectional nature of the data, which limits the ability to determine causality. Nevertheless, understanding cross-sectional associations between frailty and cognitive function is important for understanding the challenges of managing each syndrome. The lack of a criterion standard for calculating frailty is a problem common to all studies, although a definition very close to the original phenotypic definition of frailty was applied in the current study. Conceptually separating the frailty syndrome from the indicators used to define it is also difficult. The current study showed that gait speed and grip strength were specifically associated with executive function, processing speed, and attention and that individuals defined as frail had lower cognitive scores on most tests than those who were defined as prefrail or robust, suggesting that frailty defined in this way is valid for the purpose. Finally, this cohort is relatively young (mean age 61.8, range 50-93) and as such may not be representative of people who are regularly seen in geriatric clinics. This cohort will be followed up over a number of years, and future studies will determine the longitudinal outcomes of the prefrail, frail, and robust populations as they age.

In conclusion, in this cohort of individuals with a mean age in the mid-60s, there were statistically significant, albeit small, differences in cognitive function between those who were robust, prefrail, and frail as defined according to Fried's definition and independent of age, sex, education, chronic conditions, or medication use. Cognitive function is affected even in prefrailty. Phenotypic frailty and cognitive function have a complex and probably interdependent 
relationship. Small absolute effects suggest that, although a range of confounders were controlled for, residual confounding is a possibility and that longitudinal data are needed to validate the association.

\section{ACKNOWLEDGMENTS}

The authors would like to acknowledge the contribution of the participants in the study, members of the TILDA research team, study nurses, and administrators.

Funding was received from the Atlantic Philanthropies, the Irish Government, and Irish Life plc.

Conflict of Interest: The editor in chief has reviewed the conflict of interest checklist provided by the authors and has determined that the authors have no financial or any other kind of personal conflicts with this paper.

Author Contributions: Robertson, Savva: data analysis. All authors: study concept and design, preparation of manuscript.

Sponsor's Role: The sponsors had no role in the design or preparation of this manuscript.

\section{REFERENCES}

1. Crome P, Lally F. Frailty: Joining the giants. Can Med Assoc J 2011;183:889-890.

2. Heuberger RA. The frailty syndrome: A comprehensive review. J Nutr Gerontol Geriatr 2011;30:315-368.

3. Ávila-Funes JA, Amieva H, Barberger-Gateau P et al. Cognitive impairment improves the predictive validity of the phenotype of frailty for adverse health outcomes: The Three-City Study. J Am Geriatr Soc 2009;57:453461.

4. Buchman AS, Boyle PA, Wilson RS et al. Frailty is associated with incident Alzheimer's disease and cognitive decline in the elderly. Psychosom Med 2007;69:483-489.

5. Gray SL, Anderson ML, Hubbard RA et al. Frailty and incident dementia. J Gerontol A Biol Sci Med Sci 2013;68A:1083-1090.

6. Song X, Mitnitski A, Rockwood K. Nontraditional risk factors combine to predict Alzheimer disease and dementia. Neurology 2011;77:227-234.

7. Solfrizzi V, Scafato E, Frisardi V et al. Frailty syndrome and the risk of vascular dementia. The Italian Longitudinal Study on Aging. Alzheimers Dement 2013;9:113-122.

8. Aranda MP, Ray LA, Snih SA et al. The protective effect of neighborhood composition on increasing frailty among older Mexican Americans: A barrio advantage? J Aging Health 2011;23:1189-1217.

9. Raji MA, Al Snih S, Ostir GV et al. Cognitive status and future risk of frailty in older Mexican Americans. J Gerontol A Biol Sci Med Sci 2010;65A:1228-1234.

10. Robertson DA, Savva GM, Kenny RA. Frailty and cognitive impairmenta review of the evidence and causal mechanisms. Ageing Res Rev 2013;12:840-851.

11. Newman AB, Gottdiener JS, McBurnie MA et al. Associations of subclinical cardiovascular disease with frailty. J Gerontol A Biol Sci Med Sci 2001;56A:M158-M166.

12. Justin BN, Turek M, Hakim AM. Heart disease as a risk factor for dementia. Clin Epidemiol 2013;5:135-145.

13. Paulson D, Lichtenberg PA. Vascular depression: An early warning sign of frailty. Aging Ment Health 2013;17:85-93.

14. Wilson RS, Barnes LL, Mendes de Leon CF et al. Depressive symptoms, cognitive decline, and risk of $\mathrm{AD}$ in older persons. Neurology 2002;59:364-370.

15. Langlois F, Vu TT, Chasse $\mathrm{K}$ et al. Benefits of physical exercise training on cognition and quality of life in frail older adults. J Gerontol B Psychol Sci Soc Sci 2013;68B:400-404.

16. Cooper C, Li R, Lyketsos C et al. Treatment for mild cognitive impairment: systematic review. Br J Psychiatry 2013;203:255-264.
17. Langlois F, Vu TT, Kergoat MJ et al. The multiple dimensions of frailty: Physical capacity, cognition, and quality of life. Int Psychogeriatr 2012;24:1429-1436.

18. Patrick L, Gaskovski P, Rexroth D. Cumulative illness and neuropsychological decline in hospitalized geriatric patients. Clin Neuropsychol 2002;16:145-156.

19. Macuco CR, Batistoni SS, Lopes A et al. Mini-Mental State Examination performance in frail, pre-frail, and non-frail community dwelling older adults in Ermelino Matarazzo, Sao Paulo, Brazil. Int Psychogeriatr 2012;24:1725-1731.

20. Yassuda MS, Lopes A, Cachioni M et al. Frailty criteria and cognitive performance are related: Data from the FIBRA study in Ermelino Matarazzo, Sao Paulo, Brazil. J Nutr Health Aging 2012;16:55-61.

21. Boyle PA, Buchman AS, Wilson RS et al. Physical frailty is associated with incident mild cognitive impairment in community-based older persons. J Am Geriatr Soc 2010;58:248-255.

22. Avila-Funes JA, Carcaillon L, Helmer C et al. Is frailty a prodromal stage of vascular dementia? Results from the Three-City Study. J Am Geriatr Soc 2012;60:1708-1712.

23. Kearney PM, Cronin H, O'Regan C et al. Cohort profile: The Irish Longitudinal Study on Ageing. Int J Epidemiol 2011;40:877-884.

24. Fried LP, Tangen CM, Walston J et al. Frailty in older adults: Evidence for a phenotype. J Gerontol A Biol Sci Med Sci 2001;56A:M146-M156.

25. Hagstromer M, Oja P, Sjostrom M. The International Physical Activity Questionnaire (IPAQ): A study of concurrent and construct validity. Public Health Nutr 2006;9:755-762.

26. Orme JG, Reis J, Herz EJ. Factorial and discriminant validity of the Center for Epidemiological Studies Depression (CES-D) scale. J Clin Psychol 1986;42:28-33.

27. Folstein MF, Folstein SE, McHugh PR. Mini-mental state. A practical method for grading the cognitive state of patients for the clinician. J Psychiatr Res 1975;12:189-198.

28. Nasreddine ZS, Phillips NA, Bédirian V et al. The Montreal Cognitive Assessment, MoCA: A brief screening tool for mild cognitive impairment. J Am Geriatr Soc 2005;53:695-699.

29. D'Elia L. Color Trails Test: Professional Manual. Odessa, FL: Psychological Assessment Resources, 1996.

30. Robertson IH, Manly T, Andrade J et al. “Oops!” Performance correlates of everyday attentional failures in traumatic brain injured and normal subjects. Neuropsychologia 1997;35:747-758.

31. Roth M, Tym E, Mountjoy C et al. CAMDEX. A standardised instrument for the diagnosis of mental disorder in the elderly with special reference to the early detection of dementia. Br J Psychiatry 1986;149:698-709.

32. Wallace RB, Herzog AR. Overview of the health measures in the Health and Retirement Study. J Hum Resour 1995;30:84-107.

33. Rosano C, Studenski SA, Aizenstein HJ et al. Slower gait, slower information processing and smaller prefrontal area in older adults. Age Ageing 2012;41:58-64.

\section{SUPPORTING INFORMATION}

Additional Supporting Information may be found in the online version of this article:

Appendix S1. Z-scores (with standard errors) for cognitive domains among prefrail and frail older adults compared to robust, after adjusting for age, sex, education, chronic conditions, medication use and stratified by age group: 50-64, 65-74, and 75+.

Please note: Wiley-Blackwell is not responsible for the content, accuracy, errors, or functionality of any supporting materials supplied by the authors. Any queries (other than missing material) should be directed to the corresponding author for the article. 